\title{
Rational design of organelle compartments in cells
}

\author{
Claudio Angione ${ }^{\bowtie}$, Giovanni Carapezza ${ }^{2}$, Jole Costanza², Pietro Lió', Giuseppe Nicosia² \\ ' Computer Laboratory, University of Cambridge, Cambridge, United Kingdom \\ 2 Department of Mathematics and Computer Science, University of Catania, Catania, Italy
}

\section{Motivation and Objectives}

In recent years there is a growing interest in researching on mitochondria, chloroplasts and other mitochondrion-like organelles (e.g. hydrogenosomes, mitosomes and apicoplasts) because of the integrate bio-search for comorbidities-related genes, pathway dysfunctions, the energy balance in aging, inflammation and disease, and the discovery of novel factors involved in organelle division, movement, signaling and adaptation to varying environmental and pathogenic conditions.

Furthermore, there is an impressive amount of mitochondria and chloroplasts sequence data (thousands of mitochondrial sequences from many species have been sequenced) that have been used in the last ten years to derive the history of species. Notably, there are no examples of examined eukaryotes without a mitochondrion-related organelle (Shiflett and Johnson, 2010). Despite these research efforts, there is a lack of knowledge about the relationships between the organelles in a cell and its metabolism.

We aim at investigating and comparing the complexity of these organelles through a common framework that includes single- and multi-objective optimization, robustness analysis and sensitivity analysis. The possibility of multiobjective-optimization in organelles such as the mitochondrion may be related to the different tasks of maximizing the ATP or the heat, or intermediate compounds of the Krebs cycle in order to provide input for biosynthetic pathways (e.g. the amino acids synthesis).

Furthermore, rather than focusing only on networks of molecules, we think of the cell as an integrated system (Yoneda et al, 2009). Indeed, the systems biology approach, i.e. taking into account only molecular networks, misses the analysis of the organization provided by organelles. An organelle can be viewed as a functional organization of macromolecules working to accomplish essential cellular functions. Many conditions depend on a variety of environmental and other factors, and therefore cannot be fully investigated by conventional molecular-level approaches.

\section{Methods}

The cell contains many membrane-bound organelles, each specialized in one or more functions. External reactions can be thought of as links between organelles, since they involve metabolites found both in the cytoplasm and in the organelles. In our framework (Figure 1), we take into account complete models of the organelles in the cell, whose state space reflects its metabolism. The genetic algorithm underlying the optimization allows us to reach the optimal Pareto-front, i.e. to move the front towards the optimal point (e.g., maximum ATP and NADH), which is unfeasible if the two objective are negatively correlated with one another. The framework is implemented in MATLAB.

\section{Results and Discussion}

The genetic and the energy-converting networks of mitochondria and chloroplasts are descended, with little modification, from those of their ancestor bacteria. In this regard, we explore how the optimization and the Pareto front analysis can provide interesting insights into the evolutionary dynamics leading to the formation of organelle compartmentalization in the singleand multi-celled life. Furthermore, the sensitivity and robustness analyses can detect clusters of parameters corresponding to clusters of chemical reactions, which are often found in the cell and reflect the presence of different pathways or membrane-bound organelles. The interplay between optimization, sensitivity and robustness is useful not merely to reach the optimal configuration for the organelles, but also to conduct tentative analyses on their parameters.

We have applied our framework to investigate models of organelles, e.g. mitochondria and chloroplasts. In the mitochondrial model (Bazil et al, 2010), we found that the most sensitive parameters are the Hexokinase max rate and the $\mathrm{F}_{1} \mathrm{~F}_{0}$ ATP synthase activity. In the multi-objective optimization stage we analyzed the ATP-NADH 


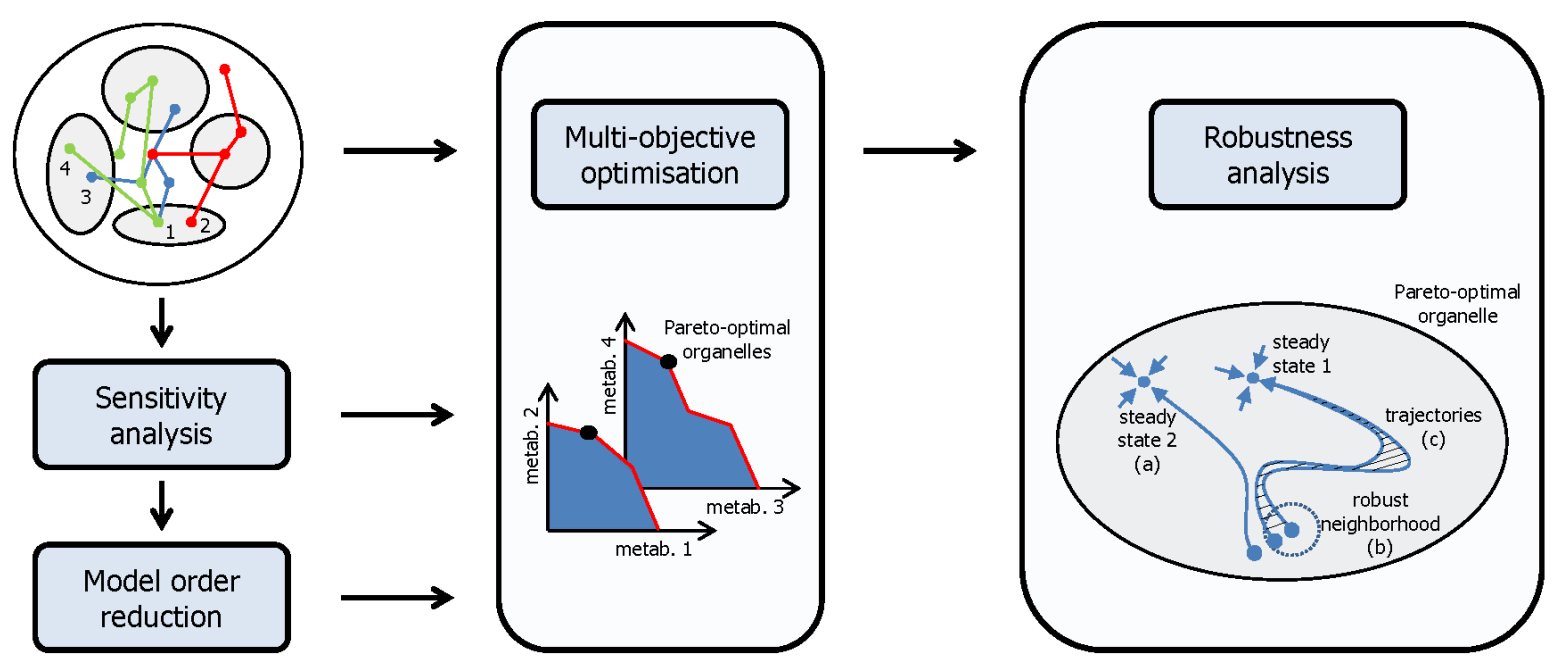

Figure 1. Framework for the rational design of the organelle network in the cell. [Left] First, we analyze the model in its highdimensional parameter space and evaluate the sensitivity of all its parameters by perturbing them in a neighborhood of the original values. The sensitivity analysis gives both insights into the role of each parameter and hints for a possible model order reduction a technique widely used to reduce the complexity of a given model. [Center] Then, we perform a multi-objective optimization on the organelle metabolism, in order to find the Pareto-optimal front involving two or more metabolites of interest (e.g., ATP and NADH). [Right] Finally, we evaluate the robustness of the Pareto-optimal solutions. Kitano has remarked on the need for a general theory of biological robustness. According to him, a system is robust if it maintains its functionality, even if it transits through a new steady state (a) or if it is unstable. According to (Stracquadanio and Nicosia, 2011), the robustness of a system is the number of robust trials over the total number of trials; a perturbation trial is said to be robust when the perturbation is in the robust neighborhood (b) such that the output remains in a given interval. According to Gunawardena, the robustness to change of initial conditions is called dynamical stability. For instance one can evaluate the differences in the dynamics of the system (c); indeed, as highlighted by (Stelling et al, 2004) robustness can also apply to dynamic processes in development.

Pareto front obtained with several calcium concentrations: if $\mathrm{Ca} 2+$ increases, we obtain an increase in NADH formation, while ATP remains constant; if $\mathrm{Ca} 2+$ drastically decreases, there is a lower ATP synthesis. Unexpectedly, with a slow decrease of $\mathrm{Ca} 2+$, both objectives are maximized. Our results highlight also that the natural mitochondrion is more robust than the optimized one, as it features a global robustness value of $26.94 \%$ and a local value of $9.00 \%$.

In the chloroplast model (Zhu et al, 2007), our framework detected RuBisCO and GAP dehydrogenase as the most sensitive enzymes of the C3 cycle. The Pareto fronts allowed us to find a trade-off between the maximization of the $\mathrm{CO}_{2}$ uptake rate and the minimization of the nitrogen consumption, with the aim of absorbing more $\mathrm{CO}_{2}$ while consuming less "leaf-fuel". RuBisCO and PGA kinase are the most robust enzymes.

The functional optimization is not partitioned or delegated to the organelles. The selection is on the phenotype and acts on the whole cell's compartmentalized genomes. Indeed, it is the whole protozoan cell that competes with other protozoa. The fact that an organelle is kept during the evolution means that its contribution to the overall protozoan's fitness is not marginal, i.e., the presence of the organelle ensures some advantages over losing it. The contribution of each organelle is both to maximize the energy production and to coevolve with the other cell structures, so as to ensure the maximum fitness of the cell. The organelles in a cell play also a key role in the neuronal degeneration. In this regard, neurotransmitters are found in vesicles, i.e. tiny organelles that allow to respond to packets ("units") of neuronal chemical signaling.

Following our framework and extending it, in the near future we plan to design, analyze and optimize the metabolism of systems composed of different species living and interacting in the same organism. In this project we have performed an in silico design that can explore the reaction network and seek in the search space the solutions that optimize two or more objectives. Therefore, our approach lies in the field of computational metabolic engineering. This kind of analysis could easily highlight the com- 
plementarity of different metabolic networks. For instance, mitochondria and chloroplasts are (usually) both found in plants, and are part of the same functional pipeline: starting from $\mathrm{CO}_{2}$, the photosynthesis in the chloroplast creates glucose that enters the mitochondria to create ATP.

The model of the whole cell of a human pathogen (Karr et al, 2012) has opened new frontiers in this research field. The whole-cell model refers to the Mycoplasma genitalium and consists of 28 submodels accounting for all the biological functions of the cell. We have already applied our methodology to Flux Balance Analysis, GeneProtein-Reaction associations, Ordinary Differential Equations (ODEs) and Differential Algebraic Equations (DAEs). Hence, our framework is suitable for general purpose or black-box analysis, enabling us to investigate not only the model of metabolism, but also the whole-cell model. Our final goal is to improve our methodology in order to tackle any BioCAD problem.

In each Pareto front we have considered the single organelle, while in the cell there are usually many organelles that could differ for activity depending on their location in the cell. In a network of organelles, most of the reactions involve more than one organelle; an appropriate approach would be to build a Pareto front where each metabolite belongs to a different organelle in the network, linking with a set of Delay Differential Equations (DDEs). DDEs differ from ODEs in that they allow rates of change to depend on the state of the system at an earlier time. In organelle systems, DDEs could account for diffusion processes and maturation events.

The integrated bio-search for a diseased, perturbed, misfunctional pathway often needs an accurate understanding of the relationships and interactions of that pathway with the organelle network system. The methodology proposed in our work could address most questions emerging in neurodegenerative and cancer disease investigation, which are focused on the interaction between organelle networks and cellular metabolism.

\section{References}

1. Bazil J N, Buzzard G T, Rundell A E (2010) Modeling mitochondrial bioenergetics with integrated volume dynamics. PLoS computational biology, 6(1):e1000632

2. Karr J R, Sanghvi J C, et al (2012) A Whole-Cell Computational Model Predicts Phenotype from Genotype. Cell, 150(2):389-401

3. Shiflett A and Johnson P J (2010) Mitochondrion-related organelles in parasitic eukaryotes. Annual review of microbiology, 64:409

4. Stelling J, et al (2004) Robustness of Cellular Functions. Cell, 118(6):675-685

5. Stracquadanio $G$ and Nicosia $G$ (2011) Computational energy-based redesign of robust proteins. Computers \& chemical engineering, 35(3):464-473

6. Yoneda $Y$, et al (2009) Frontier biomedical science underlying organelle network biology, http://www.fbs.osaka-u.ac.jp/organelle-network/eng/greeting/greeting/

7. Zhu X G, de Sturler E, Long S P (2007) Optimizing the distribution of resources between enzymes of carbon metabolism can dramatically increase photosynthetic rate. Plant Physiology, 145:513-526 